\title{
SUSY searches with the ATLAS detector
}

\author{
Riccardo Maria BIANCHI ${ }^{1}$, a , on behalf of the ATLAS Collaboration \\ ${ }^{1}$ University of Pittsburgh, Department of Physics and Astronomy, Pittsburgh PA 15260, USA
}

\begin{abstract}
Despite the absence of experimental evidence, weak scale supersymmetry remains one of the best motivated and studied Standard Model extensions. This talk summarises recent ATLAS results for searches for supersymmetric (SUSY) particles, with focus on those obtained using proton-proton collisions at a centre of mass energy of 13 $\mathrm{TeV}$. Strong production in both R-Parity conserving and R-Parity violating SUSY scenarios are considered. The searches involved final states including jets, missing transverse momentum, light leptons, as well as long-lived particle signatures.
\end{abstract}

\section{Introduction}

Supersymmetry (SUSY) postulates the existence of a twin particle for each Standard Model particle. Masses, and nature, of the supersymmetric particles depend on different parameters, addressed in many different SUSY models.

Figure 1 shows an overview of all the SUSY searches run by the ATLAS Experiment [1], which amount to about 50 analyses. In the figure, the searches are divided in categories; from the top: analyses involving strong production of squarks and gluinos; strong production involving third generation squarks (both $\tilde{g}$-mediated and from direct production); electroweak production of non-coloured SUSY partners; long-lived particles and R-parity violation decays. The light-blue bars designate the analyses that have been updated in order to analyze the experimental data taken during the Run 2 phase of the LHC operation, at a centre-of-mass energy of $13 \mathrm{TeV}$. The green bars, instead, show the mass scale explored by the analyses using the data taken in the Run 1 phase of LHC at a centre-of-mass energy of 7 and $8 \mathrm{TeV}$. All public results from the ATLAS SUSY analyses can be found at [2].

This paper presents the latest results of four searches which have been recently updated to analyze the latest available Run 2 datasets at a centre-of-mass of $13 \mathrm{TeV}$. Three of them analyze a dataset of $3.2 \mathrm{fb}^{-1}$, while the fourth exploits the latest $14 \mathrm{fb}^{-1}$ dataset.

\section{Heavy long-lived charged R-hadrons}

Gluino and squark production in proton-proton collisions can produce composite colorless states of gluinos and squarks, in addition to ordinary particles (gluon and quarks) from the Standard Model. These states are called $R$-hadrons.

At the LHC R-hadrons, if they exist, are expected to be produced as charged or neutral states, and to be heavy and slow, with a small $\beta$ velocity. They also should have a ionization energy loss $d E / d x$

\footnotetext{
a e-mail: riccardo.maria.bianchi@cern.ch
} 


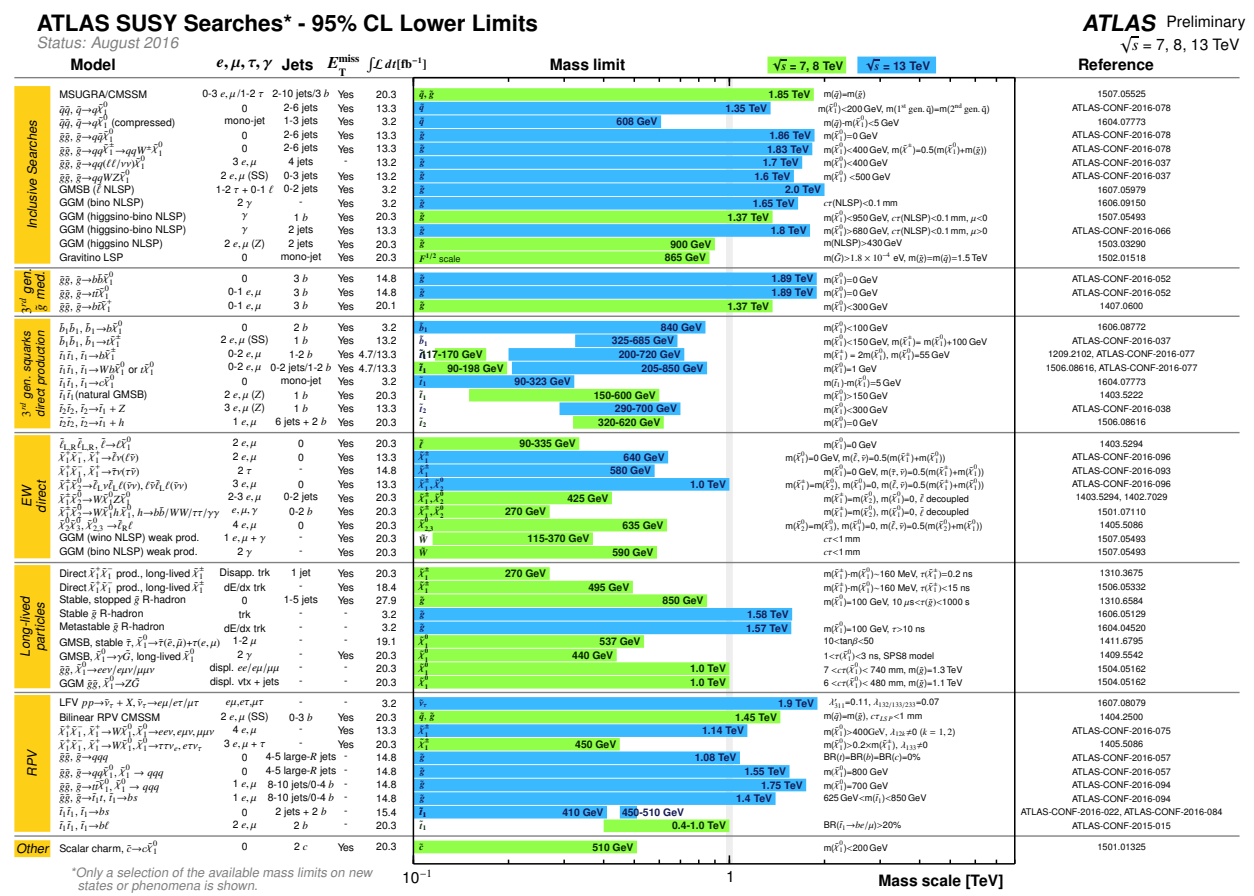

Figure 1: Mass reach of ATLAS searches for Supersymmetry [2]. Only a representative selection of the available results is shown.

larger than that of Standard Model particles. Moreover, they are expected to be able to change charge when interacting with the detector material [3, 4].

The search presented here [5] analyzes a dataset of $3.2 \mathrm{fb}^{-1}$ and makes use of information from both the pixel-based inner tracking detector and the hadronic calorimeter, in a combined way. This search extends the analysis [6], which only uses Pixel information. In this search the information from the muon spectrometer has been omitted, in order to be sensitive to scenarios when the R-hadrons decay or turn neutral before arriving in the muon system. In the search, the $d E / d x$ ratio and velocity measurements are used to infer the R-hadron mass.

\subsection{Object selection}

To tag a particle as an R-hadron candidate different criteria must be fullfilled: a very wellreconstructed isolated track, with at least 7 hits in the silicon-based tracking detector; at least 2 calorimeter clusters used to measure the $d E / d x$ ratio; a transverse momentum of the particle larger than $50 \mathrm{GeV}$ and the fullfilment of other requirements used to prevent misidentification.

Moreover, cosmic-rays are rejected by omitting a track when a similar, symmetrical track is observed in the detector. And particles from the decay of the $Z$ boson to muons are rejected by omitting reconstructed tracks whose invariant mass is in the $[81,101] \mathrm{GeV}$ window. 


\subsection{Event selection and background estimation}

For the online event selection a trigger with an $E_{\mathrm{T}}^{\text {miss }}$ signature and a cut at $70 \mathrm{GeV}$ has been used, whose signal efficiency is in the range $32 \%-50 \%$, depending on the R-hadron mass. For the offline event selection, the requirements are a primary vertex reconstructed from at least two tracks with $p_{\mathrm{T}}$ $>400 \mathrm{MeV}$, and one R-hadron candidate. Then, for the final event selection, extra requirements are imposed: a $p_{\mathrm{T}}$ above $200 \mathrm{GeV}$; a R-hadron $\beta$ velocity smaller than 0.75 and a product $\beta \gamma$ smaller than 1.35 or 1.15 , according to the R-hadron mass.

The signal region is defined in the $m_{\beta}-m_{\beta \gamma}$ plane, as shown in figure 2.

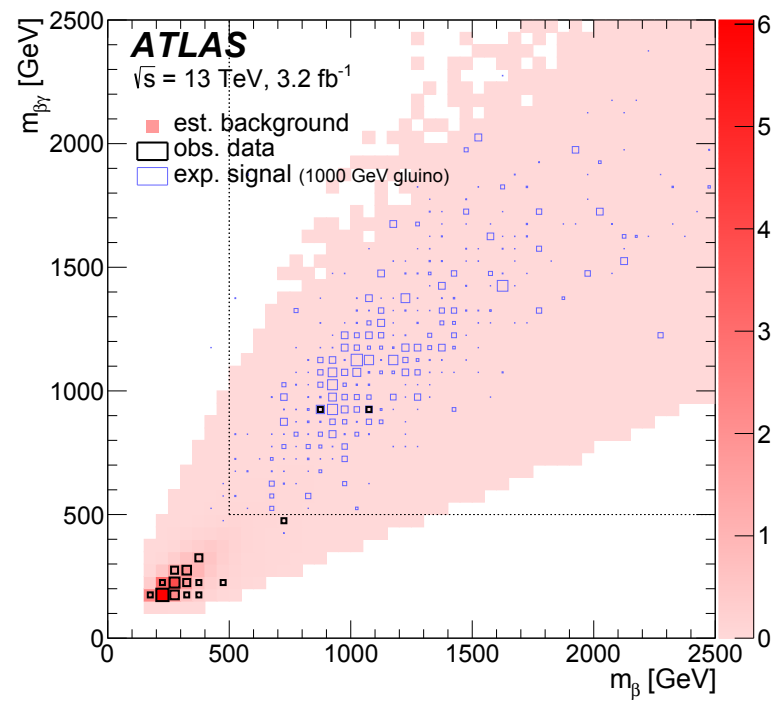

Figure 2: Data (bold boxes) and background estimates (colour fill) for $m_{\beta}$ vs. $m_{\beta \gamma}$ for the gluino R-hadron search for the $1000 \mathrm{GeV}$ mass hypothesis. The blue thin-line boxes illustrate the expected signal on top of the background. The signal region is shown by the two black dashed vertical/horizontal lines at $500 \mathrm{GeV}[5]$.

The final signal selection efficiency is of $9 \%-15 \%$ for gluino and stop R-hadrons and $6 \%-8 \%$ for sbottom R-hadrons. The lower efficiency for bottom squarks is expected, as bottom R-hadrons tend to be neutral more often than those with up-type squarks [5].

The background is evaluated in a data-driven manner, randomly picking momentum, $\beta$ and $\beta \gamma$ values sampled from probability distributions functions (PDF) determined from data; that yields mass distributions estimating the background.

\subsection{Results}

Two events with masses above $500 \mathrm{GeV}$ pass the final event selection for the $1000 \mathrm{GeV}$ mass hypothesis (figure 2), while only one of these events passes the final event selection for the $1600 \mathrm{GeV}$ mass hypothesis. However no statistically significant excess is observed in the whole analized mass range, 
and therefore $95 \%$ CL exclusion limits are placed on the R-hadron production cross section, as shown in figure 3. The cross-section limits are translated into expected lower limits at 95\% CL on R-hadron masses of $1655 \mathrm{GeV}, 865 \mathrm{GeV}$ and $945 \mathrm{GeV}$ for the production of long-lived gluino, sbottom and stop R-hadrons, respectively. Corresponding observed lower mass limits at 95\% CL for gluino, sbottom and stop are set, as well, at $1580 \mathrm{GeV}$ for the gluino mass, at $805 \mathrm{GeV}$ for the sbottom mass and at $890 \mathrm{GeV}$ for the stop mass. The figure also shows the limits set by the previous search with the data collected at a centre-of-mass energy of $8 \mathrm{TeV}$ [7], for comparison. The current search greatly improves the older limits.

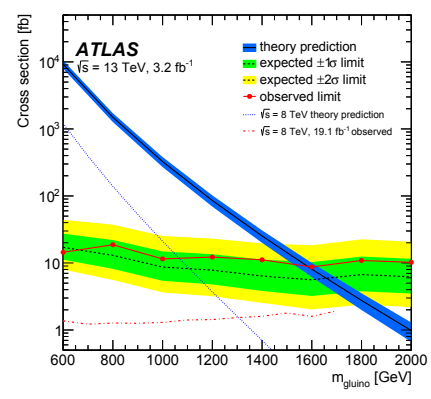

(a) gluino exclusion plot

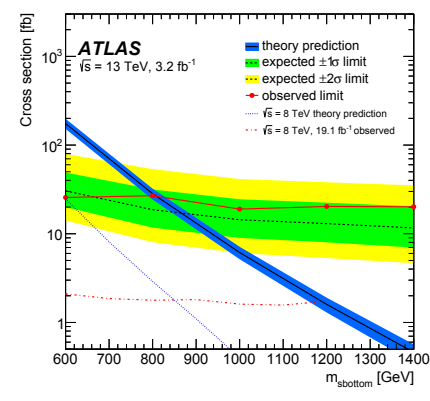

(b) sbottom exclusion plot

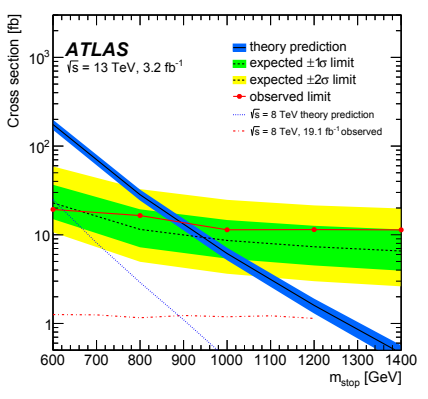

(c) stop exclusion plot

Figure 3: Exclusion plots for gluino, sbottom and stop [5].

\section{Two leptons, jets and $E_{\mathrm{T}}^{\text {miss }}$ search}

This section presents two searches for new phenomena in final states containing a same-flavour (SF) opposite-sign (OS) lepton pair (electrons or muons), jets, and large $E_{\mathrm{T}}^{\text {miss }}$ : the so-called "on-shell $Z$ " and "edge" searches [8]. The "on-shell $Z$ " channel requires a lepton pair whose invariant mass $m_{l l}$ is consistent with the mass of the $Z$ boson mass, while the "edge" search considers all SFOS lepton pairs. The requirement of two leptons in the final state suppresses large SM backgrounds from QCD multijet and $W+$ jets production.

The diagrams of the processes considered for this search are shown in figure 4. SFOS leptons can come from the $\tilde{\chi}_{2}^{0}$ decay, which depend on the mass difference $\Delta m_{X}=m_{\tilde{\chi}_{2}^{0}}-m_{\tilde{\chi}_{1}^{0}}$, the charginoneutralino mixing and on the presence of SUSY particles with masses $<m_{\tilde{\chi}_{2}^{0}}$ which can be produced in the decay.

The "on-shell Z" search targets the processes where $\Delta m_{X}>M_{Z}$, leading to a peak in the invariantmass distribution near $m_{l l} \approx m_{Z}$. The "edge" search analyzes the processes where $\Delta m_{X}<M_{Z}$, which lead to a rising $m_{l l}$ distribution truncated at a kinematic endpoint below the $\mathrm{Z}$ mass; depending on the mass difference $m_{X}$ and the mass of the sleptons produced in the decay, other kinematic endpoints can appear as well. Three models are considered for the "on-shell $Z$ " search, involving squark-pair and gluino-pair production; and two models are considered for the edge search, both involving the direct gluino-pair production, which differ by the $\tilde{\chi}_{2}^{0}$ decay mode.

SUSY signals are generated over a two-dimensional plane varying the gluino, squark and neutralino masses. All other supersymmetric particles are decoupled: they are considered having masses compatible with a much higher energy scale than that at the LHC, and thus not contributing in the analyzed processes. 


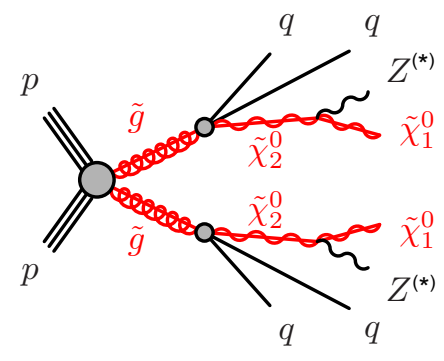

(a) "on-shell Z" channel diagram

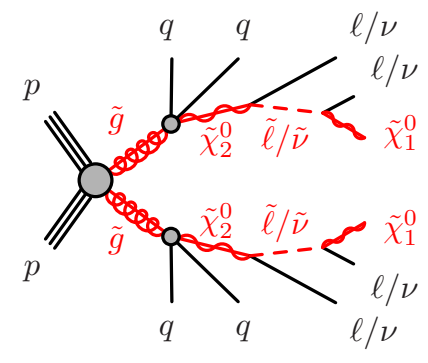

(b) "edge" channel diagram

Figure 4: The diagrams of the processes considered for the di-lepton "on-shell Z" (a) and "edge" (b) searches [8].

The search has been recently updated to exploit $14.7 \mathrm{fb}^{-1}$ of LHC $p p$ collision data at $\sqrt{s}=13$ $\mathrm{TeV}$ collected by the ATLAS detector in 2015 and 2016.

\subsection{Event selection and background estimation}

Data events are collected using a combination of single-lepton and di-lepton triggers. For both searches events are required to contain at least two signal leptons (electrons or muons) with $p_{\mathrm{T}}>$ $25 \mathrm{GeV}$, which must pass at least one of the leptonic triggers. If more leptons are present, the selection process continues based on the two leading leptons (those with the highest $p_{\mathrm{T}}$ ).

For each search signal regions (SRs) are defined to accept events from the SUSY signals, control regions (CRs) are built to be empty of SUSY events and to estimate the background events, and validation regions (VRs) are designed to help validating the SR and CR selections. Figure 5 shows all the most relevant regions defined for the two searches.

One signal region (SRZ) is defined for the "on-shell $Z$ " search, requiring a higher $p_{\mathrm{T}}$ for the leading electrons $\left(p_{\mathrm{T}}>50 \mathrm{GeV}\right)$, a large missing transverse energy $\left(E_{\mathrm{T}}^{\text {miss }}>225 \mathrm{GeV}\right)$, two or more jets, two leptons whose invariant mass is in the range [81,101] GeV, and a large $H_{T}>600 \mathrm{GeV}^{1}$.

The "edge" search requires at least two leptons with $p_{\mathrm{T}}>25 \mathrm{GeV}$, and it explores the full $m_{l l}$ spectrum, with the only exception for the region with $m_{l l}<12 \mathrm{GeV}$, rejected to suppress Drell-Yann processes and low mass resonances. For this search three SRs are defined, according to the value of the gluino-neutralino mass difference $\Delta m_{\tilde{g}}=m_{\tilde{g}}-m_{\tilde{\chi}_{1}^{0}}$, called SR-low, SR-medium and SR-high. All the three regions require $E_{\mathrm{T}}^{\text {miss }}>200 \mathrm{GeV}$, while SR-medium and SR-high also require an $H_{T}$ quantity of $400 \mathrm{GeV}$ and $700 \mathrm{GeV}$ respectively.

The SRs dominant background for this searches comes from "flavor-symmetric" (FS) processes 2 : $t \bar{t}$ (which dominates), $W W, Z Z$ and $Z \tau \tau$. Those FS processes amount to the $60 \%-90 \%$ of the total background and are estimated with a data-driven approach, using control samples of $e \mu$ events. The $Z / \gamma+$ jets contribution is small but can mimic the signal, so it is estimated with a data-driven method as well. Di-boson production ( $W Z$ and $Z Z$ ) gives a contribution of 5\%-20\% to the total background and are estimated with simulated Monte Carlo data and then validated with dedicated $3 l(W Z)$ and $4 l$

\footnotetext{
${ }^{1} H_{T}$ is the total visible transverse energy, generally defined as the scalar sum of the transverse momenta of the photons, leptons and jets in the event. For the "edge" search, leptons are not included in the $H_{T}$ definition.

${ }^{2}$ In FS processes the di-leptonic ratio is $e e: \mu \mu: e \mu=1: 1: 2$ because of the two leptons coming from independent semi-leptonic $W$ decays.
} 


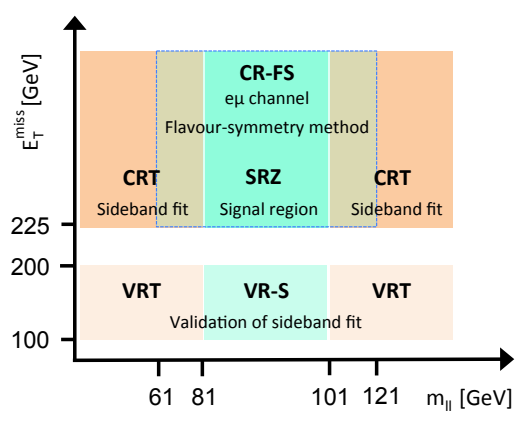

(a) "on-shell Z" channel diagram

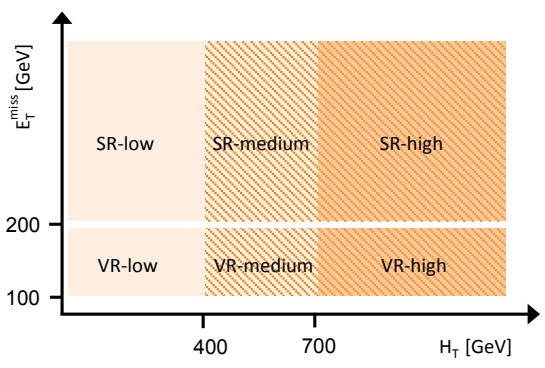

(b) "edge" channel diagram

Figure 5: Schematic diagrams of the signal, control and validation regions (SR, CR and VR respectively) for the "on-shell Z" (a) and "edge" (b) searches [8]. For the "on-shell $Z$ " search the various regions are shown in the $m_{l l^{-}}$ $E_{\mathrm{T}}^{\mathrm{miss}}$ plane, whereas in the case of the "edge" search the signal and validation regions are depicted in the $H_{T}-E_{\mathrm{T}}^{\text {miss }}$ plane.

(ZZ) validation regions (VRs). All other sources of background - like $t t W, t t W, t t W W-$ provide small contributions and are estimated with simulated data only.

\subsection{Results}

The observed yields and the expected background in the SRs for both searches are shown in figure 6. For the "on-shell $Z$ " search (figure 6a), a total of 60 events are observed in data, with a predicted background of $53.5 \pm 9.3$ events; the significance ${ }^{3}$ corresponds to $0.47 \sigma$, expressed in terms of standard deviations. For the "edge" search, since signal models may produce kinematic endpoints at any value of $m_{l l}$, a "sliding window" approach is used to define possible di-lepton mass windows. Events selected in the three signal regions are then further grouped into non-orthogonal windows based on the $m_{l l}$ value, chosen because they are the most sensitive ones for at least one grid point in the signal model parameter space. The expected and observed yields in the 24 (overlapping) $m_{l l}$ ranges of SRlow, SR-medium, and SR-high signal regions are shown in figure 6b, together with their significance.

No statistically significant excesses are observed, so exclusion limits are set. The results of the "on-shell $Z$ " search are interpreted in a simplified model with gluino-gluino pair production, where the gluinos decay as $\tilde{g} \rightarrow q \bar{q} \tilde{\chi}_{1}^{0}$ and the $\tilde{\chi}_{2}^{0}$ decays as $\tilde{\chi}_{2}^{0} \rightarrow Z \tilde{\chi}_{1}^{0}$, with the mass of $\tilde{\chi}_{1}^{0}$ set to $1 \mathrm{GeV}$. The "edge" search results are interpreted in two simplified models with gluino-gluino pair production, with gluinos decaying as $\tilde{g} \rightarrow q \bar{q} \tilde{\chi}_{2}^{0}$.

Figures 7a-7c show the exclusion plots for the "on-shell $Z$ " search, for different signal grids; while figure $7 \mathrm{~d}$ shows the exclusion plot for the "edge" search. In all figures the observed limits are shown by the solid red line, with the dotted red lines indicating the variation resulting from varying the signal cross-section within its uncertainty $\left( \pm 1 \sigma_{\text {theory }}^{\text {SUS } Y}\right.$ ). The dashed blue line indicates the expected limits at 95\% CL and the yellow band shows the $1 \sigma$ variation of the expected limit as a consequence of the uncertainties in the background prediction and the experimental uncertainties in the signal $\left( \pm 1 \sigma_{\text {exp }}\right)$.

\footnotetext{
${ }^{3}$ Significance is the probability for the background to produce a fluctuation greater than or equal to that observed in data, when expressed in terms of the number of standard deviations $\sigma$.
} 


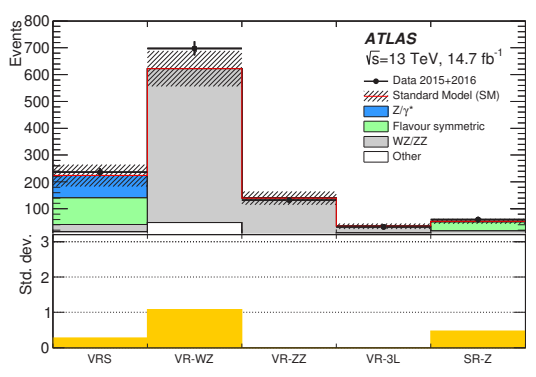

(a) "on-shell Z" search

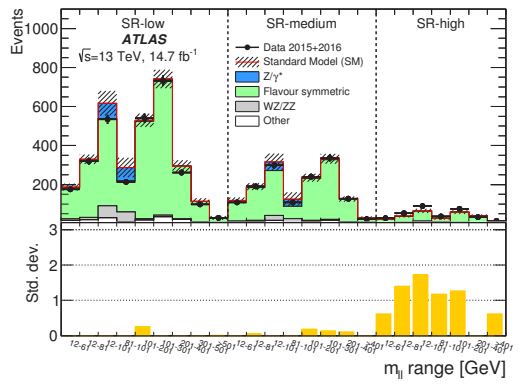

(b) "edge" search

Figure 6: The expected and observed yields in the signal regions for the "onshell Z" (a) and "edge" (b) searches [8]. The data are compared to the sum of the expected backgrounds. The significance of the difference between the data and the expected background is shown in the bottom plot; for regions in which the data yield is less than expected, the significance is set to zero. The hashed bands include the statistical and systematic uncertainties in the background prediction.

The two searches set a lower limit on the $\tilde{g}$ mass $m_{\tilde{g}}$ at $1.7 \mathrm{TeV}$, and at $980 \mathrm{GeV}$ on the $\tilde{q}$ mass $m_{\tilde{q}}$.

\section{Two photons plus $E_{\mathrm{T}}^{\text {miss }}$ search}

In this search [9] the results are interpreted in the context of general-gauge-mediation (GGM) SUSY model, with the Gravitino $\tilde{G}$ as the Lightest Supersymmetric Particle (LSP) with a very low mass $\left(m_{\tilde{G}} \ll 1 \mathrm{GeV}\right)$. In this model-dependent search the mass of the gluino $\left(m_{\tilde{g}}\right)$ and the mass of the neutralino $\left(m_{\tilde{\chi}_{1}^{0}}\right)$ are considered as free parameters, with the only constraint of the mass of the neutralino to be smaller than the gluino mass $\left(m_{\tilde{\chi}_{1}^{0}}<m_{\tilde{g}}\right)$. All other SUSY masses are considered decoupled. In the examined model, R-parity conservation is assumed, so sparticles are produced in pairs; and the bino-like neutralino $\tilde{\chi}_{1}^{0}$ is considered being the NLSP, which decays to $\tilde{G}$ plus Standard Model particles, with a high probability of photons $(\gamma+\tilde{G})$. In the end a long decay chain is expected, with two photons and missing transverse energy in the final states; the diagram is shown in figure $9 \mathrm{a}$.

\subsection{Event selection}

For the online event selection a di-photon trigger is used, with a $p_{\mathrm{T}}$ threshold of $50 \mathrm{GeV}$. For the offline event selection a signal region is defined, requiring two isolated photons with $p_{\mathrm{T}}$ larger than 75 $\mathrm{GeV}$, large missing transverse energy $\left(E_{\mathrm{T}}^{\text {miss }}>175 \mathrm{GeV}\right)$ and large effective mass $\left(m_{\mathrm{eff}}>1500 \mathrm{GeV}\right)$, defined as the scalar sum of the transverse momenta of the photons, leptons and jets in the event, plus the missing transverse energy. Additional cleaning cuts are applied to reject events from beam, cosmic rays and detector noise. A single signal region for all gluino-neutralino mass points is used, since all the mass points give very similar results. The SR has been optimized on $E_{\mathrm{T}}^{\mathrm{miss}}, m_{\mathrm{eff}}$ and $p_{\gamma}$ for two benchmark signal points, with high and low neutralino mass: $\left(m_{\tilde{g}}, m_{\tilde{\chi}_{1}^{0}}\right)=(1500,1300) \mathrm{GeV}$ and $\left(m_{\tilde{g}}, m_{\tilde{\chi}_{1}^{0}}\right)=(1500,100) \mathrm{GeV}$. 


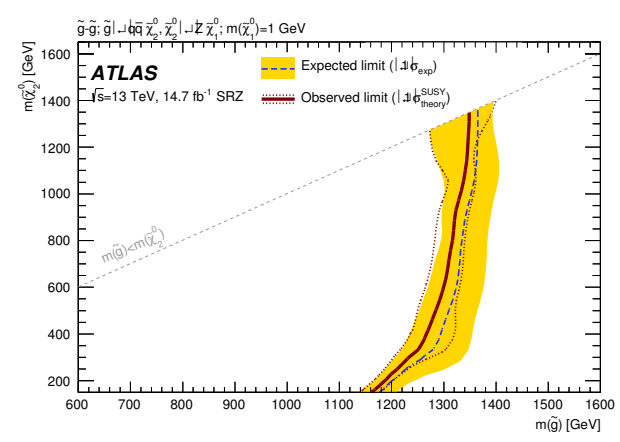

(a) "On-shell Z" $\left(\tilde{g}-\tilde{\chi}_{2}^{0}\right)$

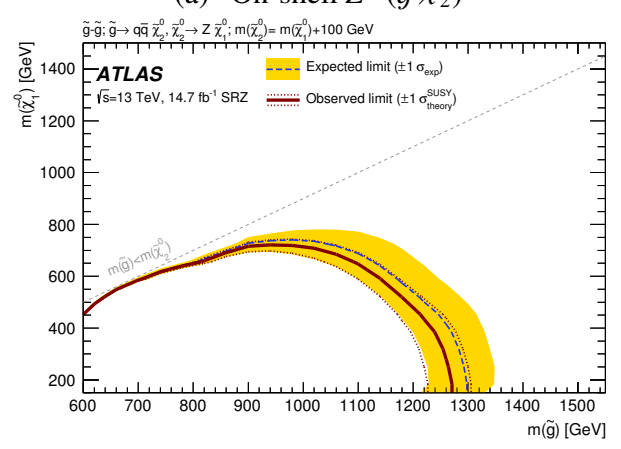

(c) “On-shell Z” $\left(\tilde{g}-\tilde{\chi}_{1}^{0}\right)$

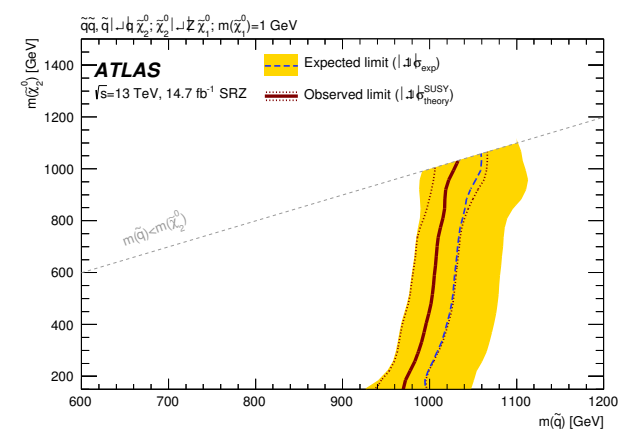

(b) "On-shell Z” $\left(\tilde{q}-\tilde{\chi}_{2}^{0}\right)$

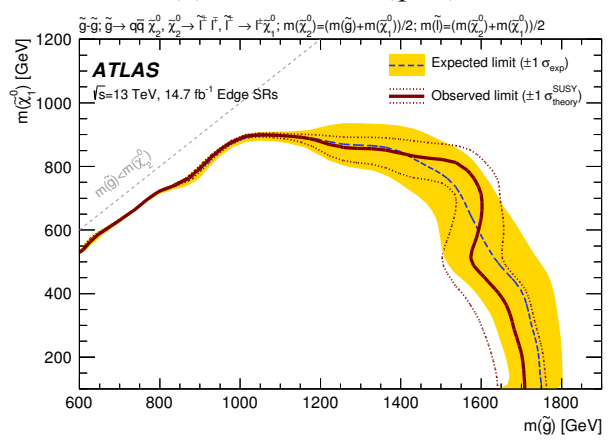

(d) "edge"

Figure 7: Exclusion plots: (a, b, c) "on-shell Z" search, for different signal grids; (d) "edge" search [8].

In figure 8 the $E_{\mathrm{T}}^{\text {miss }}$ and $m_{\mathrm{eff}}$ distributions from data and background are shown, together with those from the two SUSY signal benchmark points. The yellow band represents the uncertainty in the data/SM ratio that arises from the statisically limited estimations of the SM background.

\subsection{Background estimation}

The main sources of background for this search are: QCD processes, both from real di-photon plus jets events and from jet-faking events, estimated with a data-driven method; the electron-faking events from $W, Z$ and $t \bar{t}$ processes where an electron is misreconstructed as a photon, estimated with a datadriven method as well; irreducible background from $W \gamma \gamma$ and $Z \gamma \gamma$, estimated with simulation (the $W \gamma \gamma$, being an important contribution, is also normalized from a $l \gamma \gamma$ control region).

The total experimental uncertainty is estimated to be about $4.7 \%$ (the list of all sources can be found in [9]).

\subsection{Results}

No events are observed in the signal region, so lower limits on particle masses are set. Figure $9 \mathrm{~b}$ shows the exclusion plot for the di-photon search, varying the value of the gluino and neutralino masses in 


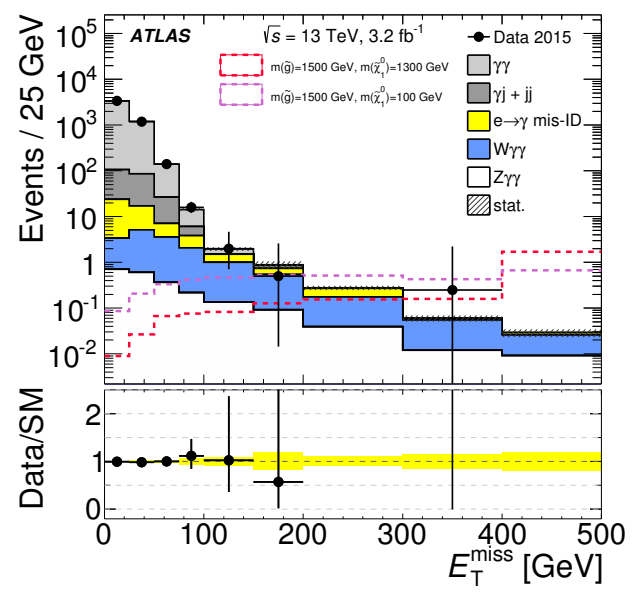

(a)

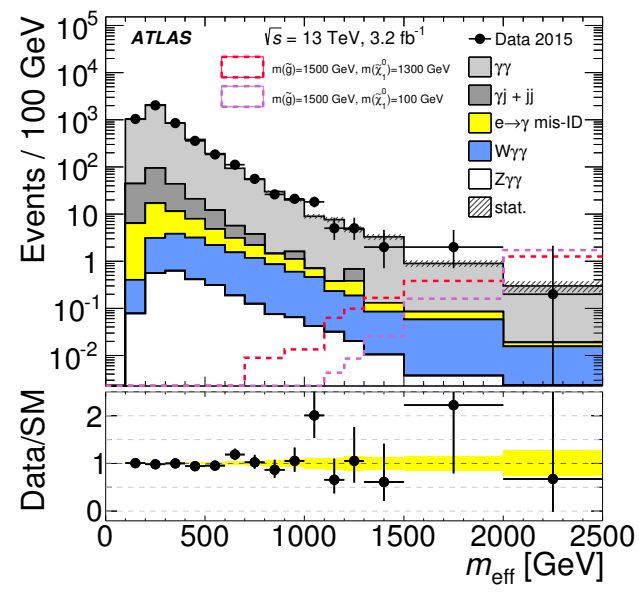

(b)

Figure 8: Distributions of $E_{\mathrm{T}}^{\text {miss }}$ (a) and $m_{\mathrm{eff}}$ (b) for the di-photon search [9].

the $m_{\tilde{g}}-m_{\tilde{\chi}_{1}^{0}}$ plane. The observed limits are shown for the nominal SUSY model cross section, as well as for a SUSY cross section increased and lowered by one standard deviation $\sigma$ of the systematic uncertainty on the process cross-section. The expected limit is also shown, together with its $\pm 1 \sigma$ range. The area previously excluded from ATLAS using $8 \mathrm{TeV}$ data [10] is also shown, in grey.

The plot shows the observed lower limit on the gluino mass as roughly independent of the binolike neutralino mass. A lower limit has been set on the mass of a GGM degenerate octet of gluino states at $1650 \mathrm{GeV}$, for a neutralino mass of $250 \mathrm{GeV}$, which extends the corresponding limit of 1340 $\mathrm{GeV}$ from the similar ATLAS search at $8 \mathrm{TeV}$.

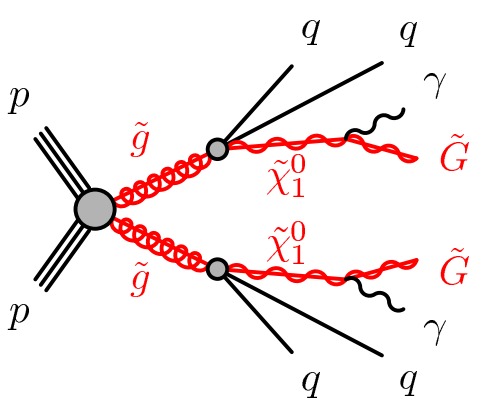

(a)

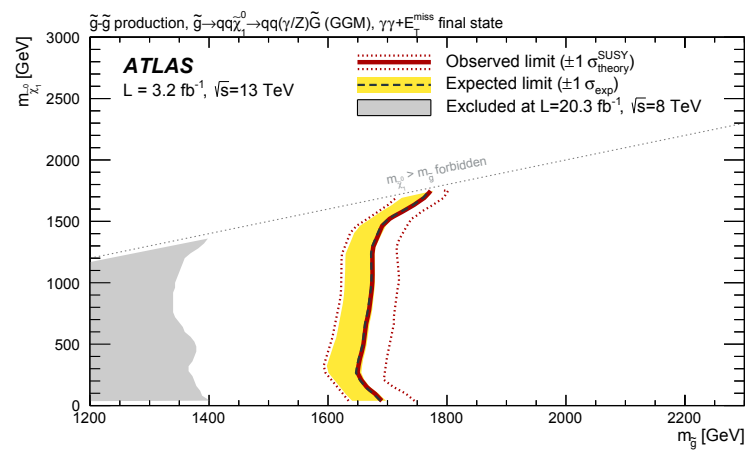

(b)

Figure 9: (a) The process considered for the di-photon plus missing transverse energy search. (b) The exclusion plot for the di-photon search [9]. 


\section{Taus plus jets plus $E_{\mathrm{T}}^{\text {miss }}$ search}

The ATLAS Collaboration recently updated the search for new physics in events with hadronicallydecaying tau leptons, jets and missing transverse momentum [11].

Two channels are considered in this search: the one containing exactly one $\tau$ lepton, and the one with 2 or more $\tau$. The results are interpreted in the context of two models: a simplified model of gluino-pair production with $\tau$-rich decay cascade (figure 10a), where the masses of the gluino and the neutralino are taken as free parameters ; and a Gauge-Mediated-Supersymmetry-Breaking (GMSB) (figure 10b), where $\Lambda$ and $\tan \beta$ are taken as free parameters.

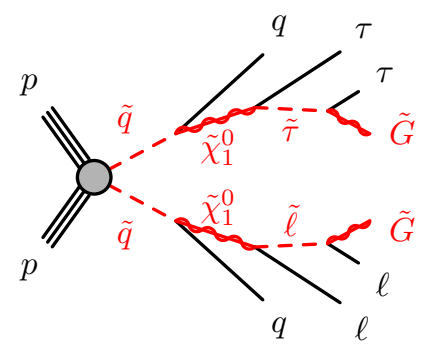

(a)

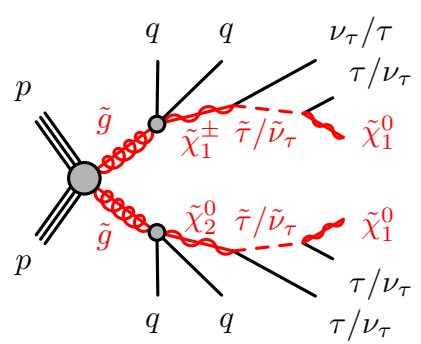

(b)

Figure 10: The processes considered in the tau search for (a) the simplified gluino-pair production model and (b) for the GMSB model [11].

\subsection{Event selection and background estimation}

For the online event selection, an $E_{\mathrm{T}}^{\text {miss }}$ trigger is used, with a threshold of $E_{\mathrm{T}}^{\text {miss }}>180 \mathrm{GeV}$. For the offline event selection, many observables are used as discriminating variables: the transverse mass $m_{l}^{T}$, the total visible transverse energy $H_{T}$ (defined as the sum of the transverse momentum of the taus plus the transverse momentum of the jets), the transverse missing energy $E_{\mathrm{T}}^{\text {miss }}$, the effective mass $m_{\text {eff }}$ (defined as the sum of $H_{T}$ and $E_{\mathrm{T}}^{\mathrm{miss}}$ ), the stransverse mass $m_{T 2}^{\tau \tau}[12,13]$, the sum of transverse masses, the total number of jets and the total number of $b$-jets.

Three signal regions (SR) are chosen for the 1- $\tau$ channel, according to different mass splittings between the gluino and the LSP (Gravitino $\tilde{G}$ or Neutralino $\left.\tilde{\chi}_{1}^{0}\right)$ : the Compressed SR $(<100 \mathrm{GeV})$, the Medium-Mass SR (500-900 GeV) and the High-Mass SR (> $1200 \mathrm{GeV})$. The Compressed SR targets topologies where a jet from initial-state radiation (ISR), and with high $p_{\mathrm{T}}$, recoils against the gluino pair whose decay products receive a Lorentz boost. Both LSPs tend to be emitted opposite to the ISR jet in the transverse plane and, therefore, such events would have large $E_{\mathrm{T}}^{\mathrm{miss}}$. The Medium-Mass and High-Mass SRs include cuts on the transverse mass, to suppress the contribution of $W(\tau v)+$ jets and of tau $t \bar{t}$ events. No SR is defined for the GMSB model, since the sensitivity of the 1- $\tau$ channel is expected to be much lower than that of the 2- $\tau$ channel.

For the 2- $\tau$ channel, two signal regions have been defined: the Compressed SR $(<900 \mathrm{GeV})$ and the High-Mass SR (> $1200 \mathrm{GeV}$ ). The Compressed SR features a cut on the stransverse mass $m_{T 2}^{\tau \tau}$ to suppress SM background contributions, which exhibit a kinematic endpoint around the $W$ and $Z$ masses. A cut on the sum of transverse masses is also applied, to take advantage of the large $E_{\mathrm{T}}^{\mathrm{miss}}$ and the large $\tau$ and jet multiplicity expected for signal events. The High-Mass SR includes a requirement 
on $H_{T}$, which is efficient for high-mass gluino signals. A signal region is also defined for the GMSB model, and targets more specifically events in which squark-antisquark production takes place, in the region $\Lambda \geq 80 \mathrm{TeV}$, which has not been not excluded by Run 1 searches.

In both channels, the dominant background originates from SM processes involving the top quark or a massive vector boson and jets. Different control and validation Regions (CRs and VRs) are defined, to help estimating the background.

\subsection{Results}

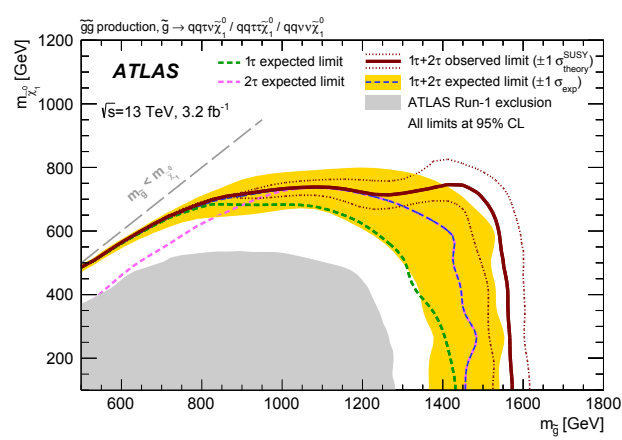

(a)

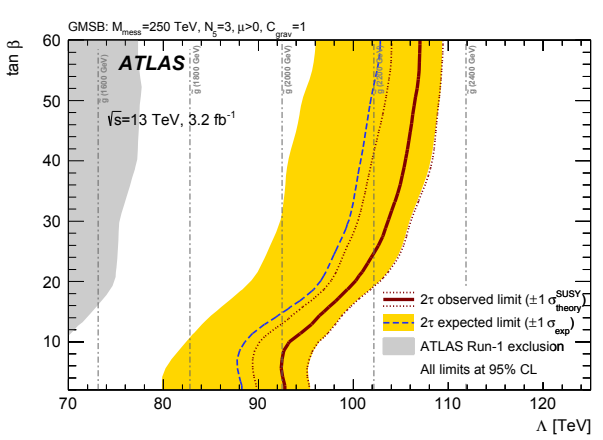

(b)

Figure 11: Exclusion contours at 95\% CL for the (a) simplified gluino-pair production model and (b) for the GMSB model [11].

No excesses are observed by this search, therefore exclusion limits are set. Figure 11 shows the exclusion plots for the two models. Figure 11a, in particular, shows the exclusion contours at 95\% CL for the simplified model of gluino-pair production, in the gluino-neutralino mass plane, based on the results from both the 1- $\tau$ and the $2-\tau$ channels, plus their combination. Figure $11 \mathrm{~b}$ shows, instead, the exclusion plot for the GMSB model, in the $\Lambda$ - $\tan \beta$ plane. In both plots, the red solid line and the blue dashed line correspond to the observed and median expected limits, respectively: for the combination of the two channels in figure 11a and for the 2- $\tau$ channel in figure 11b. The yellow band shows the one standard-deviation range of expected limits around the median value. The effect of the uncertainty on the signal cross-section is shown as a red dotted line. The grey-shaded areas in the plots show the areas previously excluded by the searches at $8 \mathrm{TeV}$, for comparison.

In the context of the simplified gluino-pair production model, the analysis sets a lower limit on the mass of the gluino $m_{\tilde{g}}$ at $1.57 \mathrm{TeV}$, for a neutralino mass $m_{\tilde{\chi}_{1}^{0}}$ around $100 \mathrm{GeV}$; and a lower limit on the mass of the neutralino $m_{\tilde{\chi}_{1}^{0}}$ at $700 \mathrm{GeV}$ for a gluino mass in the range $800 \mathrm{GeV}<m_{\tilde{g}}<1.5 \mathrm{TeV}$, and at $750 \mathrm{GeV}$ for a gluino mass of $m_{\tilde{g}} \approx 1.4 \mathrm{TeV}$. For the GMSB model, the analysis sets a lower limit on the gluino mass $m_{\tilde{g}}$ at $2.05 \mathrm{TeV}$ for small $\tan \beta$ values; and at $2.32 \mathrm{TeV}$ for larger values of $\tan \beta$.

For both models, the new exclusion limits set analyzing $3.2 \mathrm{fb}^{-1}$ of LHC collision data at $\sqrt{s}=$ $13 \mathrm{TeV}$ significantly improve the previous limits obtained by ATLAS with $20.3 \mathrm{fb}^{-1}$ of $8 \mathrm{TeV}$ data $[14,15]$. 


\section{References}

[1] ATLAS Collaboration, The ATLAS Experiment at the CERN Large Hadron Collider, JINST 3 (2008) S08003.

[2] ATLAS Collaboration, ATLAS SUSY Results, https:/twiki.cern.ch/twiki/bin/view/AtlasPublic/ SupersymmetryPublicResults.

[3] A. C. Kraan, Interactions of heavy stable hadronizing particles, Eur. Phys. J. C 37 (2004) 91-104, arXiv:hep-ex/0404001.

[4] R. Mackeprang and D. Milstead, An updated description of heavy-hadron interactions in GEANT4, Eur. Phys. J. C 66 (2010) 493-501, arXiv:0908.1868 [hep-ph].

[5] ATLAS Collaboration, Search for heavy long-lived charged R-hadrons with the ATLAS detector in $3.2 \mathrm{fb}$ - 1 of proton-proton collision data at $\sqrt{s}=13 \mathrm{TeV}$, Physics Letters B (2016), pp. 647-665, doi: 10.1016/j.physletb.2016.07.042, arXiv: 1606.05129.

[6] ATLAS Collaboration, Search for metastable heavy charged particles with large ionization energy loss in pp collisions at $\sqrt{s}=13 \mathrm{TeV}$ using the ATLAS experiment, arxiv: 1604.04520.

[7] ATLAS Collaboration, Searches for heavy long-lived charged particles with the ATLAS detector in proton-proton collisions at $\sqrt{s}=8 \mathrm{TeV}$, JHEP 01 (2015) 068, arXiv:1411.6795 [hep-ex].

[8] ATLAS Collaboration, Search for new phenomena in events containing a same-flavour oppositesign dilepton pair, jets, and large missing transverse momentum in $\sqrt{s}=13 \mathrm{TeV}$ pp collisions with the ATLAS detector, arXiv: 1611.05791, submitted to Eur. Phys. J. C.

[9] ATLAS Collaboration, Search for supersymmetry in a final state containing two photons and missing transverse momentum in $\sqrt{s}=13 \mathrm{TeV}$ pp collisions at the LHC using the ATLAS detector, arXiv: 1606.09150, accepted by Eur. Phys. J. C.

[10] ATLAS Collaboration, Search for photonic signatures of gauge-mediated supersymmetry in 8 TeV pp collisions with the ATLAS detector, Phys. Rev. D 92 (2015) 072001, arXiv:1507.05493 [hep-ex].

[11] Aaboud, M., Aad, G., Abbott, B. et al. (ATLAS Collaboration), Search for squarks and gluinos in events with hadronically decaying tau leptons, jets and missing transverse momentum in proton-proton collisions at $\sqrt{s}=13 \mathrm{TeV}$ recorded with the ATLAS detector, Eur. Phys. J. C (2016) 76: 683, doi:10.1140/epjc/s10052-016-4481-2.

[12] C. G. Lester and D. J. Summers, Measuring masses of semiinvisibly decaying particles pair produced at hadron colliders, Phys. Lett. B463 (1999) 99-103, arXiv:hep-ph/9906349 [hep-ph].

[13] C. G. Lester and B. Nachman, Bisection-based asymmetric MT 2 computation: a higher precision calculator than existing symmetric methods, JHEP 03 (2015) 100, arXiv:1411.4312 [hep-ph].

[14] ATLAS Collaboration, Search for supersymmetry in events with large missing transverse momentum, jets, and at least one tau lepton in $20 \mathrm{fb}^{-1}$ of $\sqrt{\mathrm{s}}=8 \mathrm{TeV}$ proton-proton collision data with the ATLAS detector, JHEP 1409 (2014) 103, arXiv:1407.0603 [hep-ex].

[15] ATLAS Collaboration, Summary of the searches for squarks and gluinos using $\sqrt{s}=8 \mathrm{TeVpp}$ collisions with the ATLAS experiment at the LHC, JHEP 1510 (2015) 054, arXiv:1507.05525 [hepex]. 